\title{
A urine-based DNA methylation assay, ProCUrE, to identify clinically significant prostate cancer
}

\author{
Fang Zhao ${ }^{1,2}$, Ekaterina Olkhov-Mitsel ${ }^{1,2}$, Shivani Kamdar ${ }^{1,2}$, Renu Jeyapala', Julia Garcia', Rachel Hurst ${ }^{3}$, \\ Marcelino Yazbek Hanna ${ }^{4}$, Robert Mills ${ }^{3}$, Alexandra V. Tuzova ${ }^{5}$, Eve O'Reilly ${ }^{5}$, Sarah Kelly ${ }^{5}$, Colin Cooper ${ }^{3}$, The \\ Movember Urine Biomarker Consortium, Daniel Brewer ${ }^{3,6}$, Antoinette S. Perry ${ }^{5}$, Jeremy Clark ${ }^{3}$, Neil Fleshner ${ }^{7}$ \\ and Bharati Bapat ${ }^{1,2,7^{*}}$
}

\begin{abstract}
Background: Prevention of unnecessary biopsies and overtreatment of indolent disease remains a challenge in the management of prostate cancer. Novel non-invasive tests that can identify clinically significant (intermediate-risk and high-risk) diseases are needed to improve risk stratification and monitoring of prostate cancer patients. Here, we investigated a panel of six DNA methylation biomarkers in urine samples collected post-digital rectal exam from patients undergoing prostate biopsy, for their utility to guide decision making for diagnostic biopsy and early detection of aggressive prostate cancer.

Results: We recruited 408 patients in risk categories ranging from benign to low-, intermediate-, and high-risk prostate cancer from three international cohorts. Patients were separated into 2/3 training and 1/3 validation cohorts. Methylation biomarkers were analyzed in post-digital rectal exam urinary sediment DNA by quantitative MethyLight assay and investigated for their association with any or aggressive prostate cancers.

We developed a Prostate Cancer Urinary Epigenetic (ProCUrE) assay based on an optimal two-gene (HOXD3 and GSTP1) LASSO model, derived from methylation values in the training cohort, and assessed ProCUrE's diagnostic and prognostic ability for prostate cancer in both the training and validation cohorts.

ProCUrE demonstrated improved prostate cancer diagnosis and identification of patients with clinically significant disease in both the training and validation cohorts. Using three different risk stratification criteria (Gleason score, D'Amico criteria, and CAPRA score), we found that the positive predictive value for ProCUrE was higher (59.4-78\%) than prostate specific antigen (PSA) (38.2-72.1\%) for all risk category comparisons. ProCUrE also demonstrated additive value to PSA in identifying GS $\geq 7$ PCa compared to PSA alone (DeLong's test $p=0.039$ ), as well as additive value to the PCPT risk calculator for identifying any PCa and GS $\geq 7$ PCa (DeLong's test $p=0.011$ and 0.022 , respectively).

Conclusions: ProCUrE is a promising non-invasive urinary methylation assay for the early detection and prognostication of prostate cancer. ProCUrE has the potential to supplement PSA testing to identify patients with clinically significant prostate cancer.
\end{abstract}

Keywords: Prostate cancer, PSA, Urine, DNA methylation, Biomarker, Early detection, Overtreatment

\footnotetext{
* Correspondence: bapat@lunenfeld.ca

'Lunenfeld-Tanenbaum Research Institute, Sinai Health System, Toronto,

Canada

${ }^{2}$ Department of Laboratory Medicine \& Pathobiology, University of Toronto,

Toronto, Canada

Full list of author information is available at the end of the article
}

(c) The Author(s). 2018 Open Access This article is distributed under the terms of the Creative Commons Attribution 4.0 International License (http://creativecommons.org/licenses/by/4.0/), which permits unrestricted use, distribution, and reproduction in any medium, provided you give appropriate credit to the original author(s) and the source, provide a link to the Creative Commons license, and indicate if changes were made. The Creative Commons Public Domain Dedication waiver (http://creativecommons.org/publicdomain/zero/1.0/) applies to the data made available in this article, unless otherwise stated. 


\section{Introduction}

The introduction of circulating prostate specific antigen (PSA) test has increased the rate of diagnosis of prostate cancer (PCa) by as much as $50 \%$. However, the majority of PCa patients diagnosed through PSA screening present with low-risk, localized, Gleason score (GS) 6 tumors. Although PSA has a high negative predictive value (NPV) for $\mathrm{PCa}$, its lack of specificity, limited impact on reducing morbidity, and the harms of over-diagnosing indolent disease have raised concerns about PSA screening [1].

To reduce overtreatment and associated morbidity, the U.S. Preventive Services Task Force (USPSTF) recently recommended against PSA screening to prevent unnecessary biopsies of "clinically insignificant" PCa (CI-PCa) which included patients with benign and low-risk disease [1]. However, following these recommendations, there was a substantial decrease (42.9\%) [2] in the detection of GS $\geq 7$ disease, indicating the reduction in PSA screening could delay diagnosis of "clinically significant" $\mathrm{PCa}$ (CS-PCa) consisting of intermediateand high-risk disease. The revised recommendations now include advising men under 70 about the potential benefits and limitations of PSA based screening. However, their impact on the diagnosis of CS-PCa is currently unknown.

Several nomograms have been developed to estimate $\mathrm{PCa}$ aggressiveness following biopsy, such as the well-established D'Amico criteria [3] which includes PSA, GS, and clinical T stage. Due to the limited number of variables, patients with the same D'Amico risk category may have vastly different outcomes. Alternatively, the recently developed UCSF-Cancer of the Prostate Risk Assessment (CAPRA) score [4] is more informative due to ease of calculation and inclusion of key clinical variables including age, PSA, percent of cores positive in biopsy (\%core), clinical $\mathrm{T}$ stage, and Gleason patterns. There is also the Prostate Cancer Prevention Trial (PCPT) PCa risk calculator [5], which takes into account ethnicity, family history, PSA, age, and digital rectal exams (DREs) results to calculate the risk of finding any cancer or high-risk $(G S \geq 7)$ cancer upon biopsy. These nomograms are used to distinguish low-risk versus high-risk PCa patients for management decisions after biopsy.

Low-risk PCa patients may be recommended enrollment into an active surveillance (AS) protocol where they are monitored with DREs, PSA tests, multiparametric (mp) MRI where available, and periodic biopsies instead of definitive treatment [6]. Although AS is a preferable management option for patients with CI-PCa, many AS patients with indolent tumors still undergo additional unnecessary biopsies and suffer associated morbidities.
Consequently, there is an urgent need to develop non-invasive biomarkers to complement PSA screening for the early identification of aggressive $\mathrm{PCa}$ and to guide decision making for initial diagnostic prostate biopsy or repeat biopsies of low-risk patients on AS. To address this, the Movember foundation introduced the Global Action Plan (GAP) 1: Urine biomarker initiative, which brought together 12 research teams from seven different countries. Our study, as part of this initiative, investigated non-invasive DNA methylation biomarkers for improved prognostication of PCa.

Aberrant DNA methylation is a hallmark of $\mathrm{PCa}$ $[7,8]$. Tumor-specific gene methylation alterations are ideal biomarkers due to their stability and ease of detection from patient samples with limited amounts of DNA such as urinary sediments. Detection of DNA methylation biomarkers in urine sediment is non-invasive and may be able to supplement PSA screening to identify CS-PCa patients.

We have previously discovered and/or characterized tumor-specific DNA methylation of six genes $(A P C$, GSTP1, HOXD3, KLK10, TBX15, and TGF 2 2) in radical prostatectomy tumor samples [9-11]. Increased methylation of these genes was found to be associated with higher GS and adverse clinical prognosis. We also examined these biomarkers in post-DRE urine samples from a Canadian AS PCa patient cohort [12]. In the current study, we investigated the utility of these urinary DNA methylation biomarkers for diagnosis and prognostication of CS-PCa in three international patient cohorts.

\section{Results}

\section{Cohort characteristics}

The clinicopathologic characteristics for patient cohorts are summarized in Table 1. To mitigate any inherent biases in patient recruitment, all patients were combined, randomized, and separated into training ( $2 / 3$ of patients) and validation (1/3 of patients) cohorts $[13,14]$ (Table 2).

Age and PSA were significantly correlated with each other, as well as prostate volume, and \%core. (Additional file 1: Table S1, Spearman's $\rho p<0.01$ ).

\section{Detection of urinary DNA methylation biomarkers and association with clinicopathologic variables}

We assessed DNA methylation of our panel of biomarkers in the urinary sediment of patients recruited. Methylation frequencies (patients with percent methylated of reference (PMR) >0) ranged from 39.5\% (161/ 408 patients) for GSTP1 to $92.6 \%$ (378/408 patients) for HOXD3. PMR distribution for individual markers among benign and PCa patients is shown in Fig. 1. Five of the six gene methylation showed significant increase in $\mathrm{PCa}$ 
Table 1 Clinical characteristics from the University of East Anglia, UK (UEA), GU Biobank at UHN, Canada (UHN), Trinity College at Dublin, Ireland (Dublin)

\begin{tabular}{|c|c|c|c|c|c|c|}
\hline \multirow{2}{*}{\multicolumn{4}{|c|}{ College at Dublin, Ireland (Dublin) }} & \\
\hline & & & & Patient clinical characteristics & Training & Validation \\
\hline Patient clinical characteristics & UEA & UHN & Dublin & $n(\%)$ & $268(65.4)$ & $140(34.6)$ \\
\hline$n(\%)$ & $194(48)$ & $155(38)$ & $59(14)$ & Benign & $123(44)$ & $59(41)$ \\
\hline Benign & $109(56)$ & $46(30)$ & $27(46)$ & PCa & $145(52)$ & $81(57)$ \\
\hline PCa & $85(44)$ & $109(70)$ & $32(54)$ & Gleason score & & \\
\hline Gleason score & & & & 6 & $60(41)$ & $36(44)$ \\
\hline 6 & $17(20)$ & $64(59)$ & $15(47)$ & 7 & $55(38)$ & $28(35)$ \\
\hline 7 & $42(49)$ & $32(29)$ & $9(28)$ & $8-10$ & $30(21)$ & $17(21)$ \\
\hline $8-10$ & $26(31)$ & $13(12)$ & $8(25)$ & Clinical T stage & & \\
\hline Clinical T stage & & & & $\mathrm{T} 1$ & $101(70)$ & $48(59)$ \\
\hline $\mathrm{T} 1$ & $38(45)$ & $91(83)$ & $20(63)$ & $\mathrm{T} 2$ & $25(17)$ & $16(20)$ \\
\hline $\mathrm{T} 2$ & $14(16)$ & $16(15)$ & $11(34)$ & T3 & $11(8)$ & $11(14)$ \\
\hline T3 & $19(22)$ & $2(2)$ & $1(3)$ & $\mathrm{T} 4$ & $8(6)$ & $6(7)$ \\
\hline T4 & $14(16)$ & 0 & 0 & $\%$ Biopsy cores positive for $\mathrm{PCa}$ & & \\
\hline \% Biopsy cores positive for PCa & & & & Median & $29 \%$ & $33 \%$ \\
\hline Median & $57 \%$ & $20 \%$ & $21 \%$ & Range & $5-100 \%$ & $5-100 \%$ \\
\hline Range & $7-100 \%$ & $5-100 \%$ & $6-100 \%$ & Interquartile range & $13 \%-55 \%$ & $14 \%-63 \%$ \\
\hline Interquartile range & $33 \%-100 \%$ & $9 \%-38 \%$ & $13 \%-43 \%$ & N/A & 8 & 6 \\
\hline N/A & 9 & 1 & 0 & Age at enrollment & & \\
\hline Age at enrollment & & & & Median & 66 & 66 \\
\hline Median & 67 & 64 & 65 & Range & $42-85$ & $37-85$ \\
\hline Range & $42-85$ & $37-83$ & $46-80$ & Interquartile range & $59-71$ & $59-72$ \\
\hline Interquartile range & $62-73$ & $57-69$ & $58-71$ & PSA at presentation & & \\
\hline PSA at presentation & & & & Median & 6.9 & 7.04 \\
\hline Median & 8.4 & 5.8 & 5.9 & Range & $0.01-248$ & $0.04-377.00$ \\
\hline Range & $0.2-277.3$ & $0.01-67.31$ & $0.5-248$ & Interquartile range & $4.55-10.36$ & $4.79-11$ \\
\hline Interquartile range & $5.8-12.2$ & $3.97-9.07$ & $3.85-8.64$ & Prostate volume & & \\
\hline Prostate volume & & & & Median & 7 & 49 \\
\hline Median & 59.54 & 47 & & Range & $18.0-121.6$ & $18.0-121.6$ \\
\hline Range & $21.08-244.6$ & $16.05-127.0$ & & Interquartile range & $39.15-68.68$ & $37-65.1$ \\
\hline Interquartile range & $42.52-86.52$ & $34-57$ & & N/A & 114 & 58 \\
\hline N/A & 92 & 18 & 59 & Perineural invasion & & \\
\hline Perineural invasion & & & & Yes & 30 & 13 \\
\hline Yes & 28 & 11 & 4 & No & 97 & 55 \\
\hline No & 80 & 17 & 55 & N/A & 153 & 75 \\
\hline $\mathrm{N} / \mathrm{A}$ & 86 & 127 & 0 & CAPRA risk & & \\
\hline CAPRA risk & & & & CAPRA low & $47(32)$ & $33(41)$ \\
\hline CAPRA low & $10(12)$ & $57(52)$ & $13(41)$ & CAPRA intermediate & $56(39)$ & $26(32)$ \\
\hline CAPRA intermediate & $32(38)$ & $36(33)$ & $14(44)$ & CAPRA high & $42(29)$ & $22(27)$ \\
\hline CAPRA high & $43(51)$ & $16(15)$ & $5(16)$ & D’Amico risk & & \\
\hline D'Amico risk & & & & D'Amico low & $41(28)$ & $28(35)$ \\
\hline D'Amico low & $8(9)$ & $52(48)$ & $9(28)$ & D'Amico intermediate & $57(39)$ & $24(30)$ \\
\hline D'Amico intermediate & $29(34)$ & $39(36)$ & $13(41)$ & D'Amico high & $47(32)$ & $29(36)$ \\
\hline
\end{tabular}

Table 2 Cohort characteristics of the training and validation cohorts 


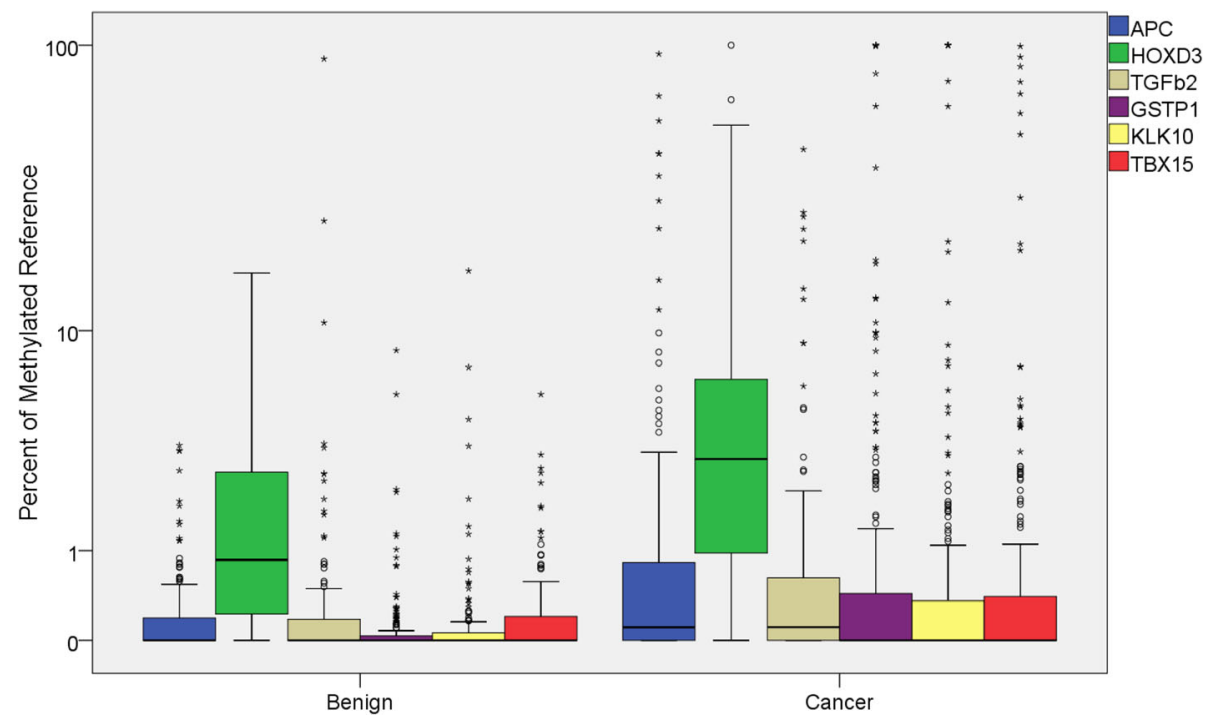

Fig. 1 Distribution of percent of methylated reference (PMR) values for individual biomarkers among benign and PCa (Cancer) patients. Number of patients $=408$. APC, HOXD3, TGFB2, GSTP1, and KLK10 are able to significantly differentiate benign and PCa (Mann Whitney Up<0.05). Circles indicate outliers within 1.5 $\mathrm{IQR}$, stars indicate outliers > 1.5 $\mathrm{IQR}$. Mann Whitney U p values can be found in Additional file 1: Table S2

compared to benign (Additional file 1: Table S2 Mann Whitney $U p<0.05)$.

Methylation levels of all six genes were significantly correlated with each other (Spearman's $\rho p<0.01$ ). $A P C$, GSTP1, KLK10, TBX15, and TGFB2 showed significant association with age and \%core (Additional file 1: Table S1; Spearman's $\rho p<0.05)$. Additionally, GSTP1, KLK10, and TBX15 were associated with PSA (Spearman's $\rho p<$ $0.05)$. HOXD3 did not correlate with any clinical variables.

\section{Building an optimal predictor gene model and ProCUrE assay}

To investigate whether combinations of biomarkers were more informative compared to individual markers for detection of any $\mathrm{PCa}$ and/or aggressive $\mathrm{PCa}$, we applied least absolute shrinkage and selection operator (LASSO) and constructed an optimal two-gene (HOXD3 and GSTP1) classifier model (ProCUrE) in the training cohort comparing between benign vs CAPRA-HR patients. Receiver operating characteristic (ROC) curve analysis of ProCUrE showed an area under curve (AUC) of 0.795 (bootstrapped 1000 iterations) (Fig. 2), which was higher than any individual marker; thus, we did not analyze individual markers in the validation cohort. An optimal cut-off threshold for ProCUrE was established with the maximum combined sensitivity (57.1\%) and specificity (97\%). Patients with methylation levels above this threshold are considered positive for ProCUrE status (ProCUrE +ve).

\section{Assessment of ProCUrE for improved PCa diagnosis}

To determine ProCUrE's value for $\mathrm{PCa}$ diagnosis, we tested its association with PCa. ProCUrE + ve status was significantly associated with $\mathrm{PCa}$ positive biopsies in both the training (Additional file 2: Figure S1A) and validation cohorts (Fig. 3a) $\quad\left(x^{2} \quad p<0.01\right)$ while age-adjusted PSA (see definition in the "Material and methods" section) $[15,16]$ was not (Fig. 3a; $\chi^{2} p>$ 0.05). ProCUrE status identified $31.6 \%$ PCa patients with $11.9 \%$ false positive cases, while age-adjusted PSA detected $75.3 \%$ PCa patients but also had a high number $(69.5 \%)$ of false positives. The positive predictive value (PPV) for ProCUrE was higher than for age-adjusted PSA (78.1\% vs 59.8\%) (Table 3A). These results demonstrate that ProCUrE +ve patients are more likely to harbor PCa.

\section{Assessment of ProCUrE for early prognostication of PCa}

To investigate ProCUrE's value for $\mathrm{PCa}$ prognostication, we assessed the ability of individual markers, ProCUrE, and clinical variables to differentiate CI-PCa and CS-PCa patients as determined by GS. Using univariable logistic regression analysis, ProCUrE, PSA, and age showed significant association with CS-PCa. Due to the difference in range of each variable, interquartile range odds ratios (IQR OR) were estimated. The IQR OR of ProCUrE in the validation cohort $(\mathrm{OR}=1.58,95 \% \mathrm{CI}=$ $1.28-1.96)$ were of similar size to PSA $(\mathrm{OR}=1.98,95 \%$ $\mathrm{CI}=1.46-2.68)$, and age $(\mathrm{OR}=1.66,95 \% \mathrm{CI}=1.13-2.45)$ for CS-PCa (Table 4A). Multivariable logistic regression of significant variables age, PSA, and ProCUrE showed that ProCUrE was an independently significant variable for CS-PCa in the validation cohort (Table 4B). These results show that ProCUrE is a robust prognosticator of CS-PCa. 


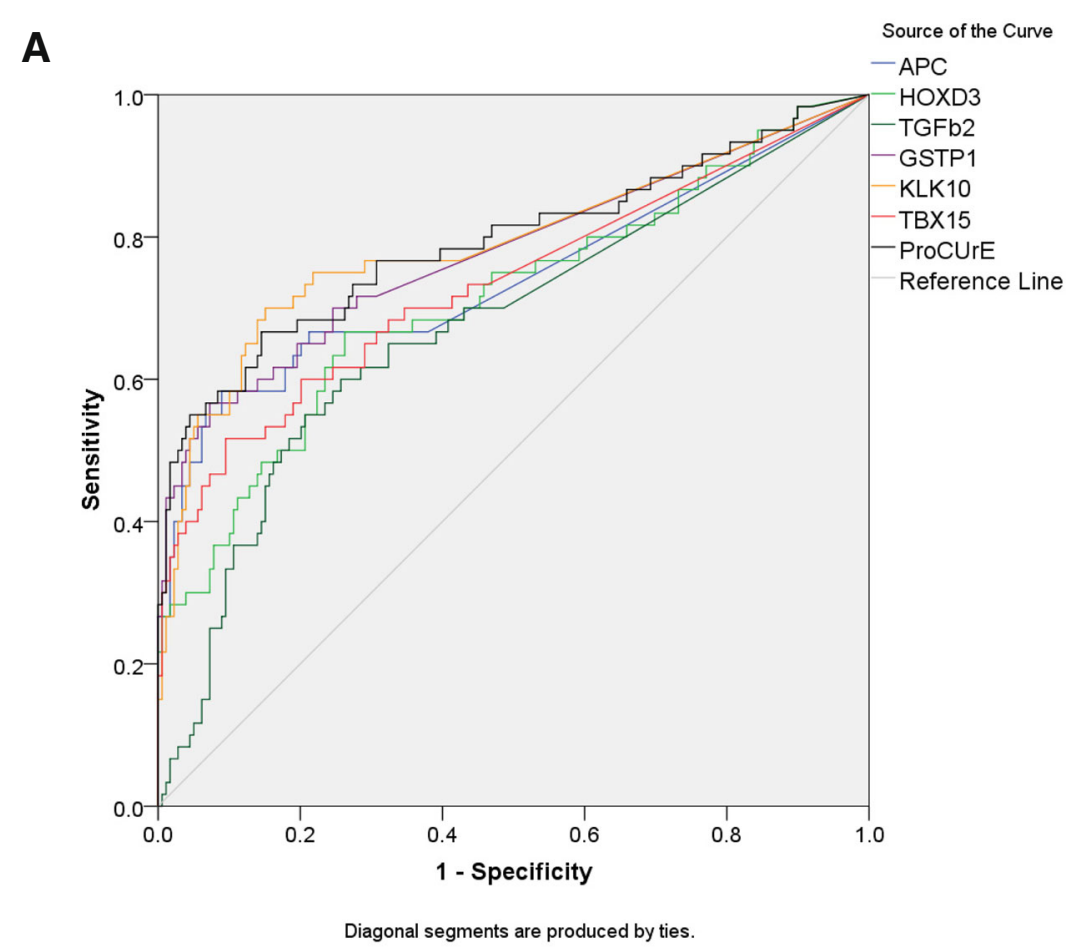

B

\begin{tabular}{llllll}
\hline & AUC & \multicolumn{2}{c}{$95 \%$ C.I. } & Sensitivity & Specificity \\
\hline ProCUrE & $0.795^{* * *}$ & 0.758 & 0.891 & $57.1 \%$ & $97.0 \%$ \\
APC PMR & $0.702^{* *}$ & 0.664 & 0.813 & $57.5 \%$ & $87.9 \%$ \\
HOXD3 PMR & $0.724^{* *}$ & 0.685 & 0.834 & $65.0 \%$ & $75.8 \%$ \\
TGFB2 PMR & 0.622 & 0.580 & 0.738 & $52.5 \%$ & $78.8 \%$ \\
GSTP1 PMR & $0.747^{* *}$ & 0.715 & 0.840 & $52.5 \%$ & $93.9 \%$ \\
KLK10 PMR & $0.749^{* * *}$ & 0.713 & 0.856 & $70.0 \%$ & $81.8 \%$ \\
TBX15 PMR & 0.645 & 0.604 & 0.767 & $40.0 \%$ & $93.9 \%$ \\
\hline
\end{tabular}

Fig. 2 a Receiver operating characteristic (ROC) curves for individual biomarkers and ProCUrE, stratifying between benign ( $n=123$ ) and CAPRA high-risk $(n=42)$ patients in the training cohort. PSA was not included in this figure since PSA is used to calculate CAPRA and will always have a very strong association with CAPRA high risk. b AUC (bootstrapped 1000 iterations), sensitivity, and specificity for each gene and ProCUrE. ROC ${ }^{* *} p<0.01 ;{ }^{* * *} p<0.001$

We examined ProCUrE among patients stratified into different risk categories based on GS, D'Amico criteria and CAPRA score. $\chi^{2}$ analysis showed that both ProCUrE and age-adjusted PSA could differentiate between patients harboring no disease and/or CI-PCa versus $\mathrm{CS}-\mathrm{PCa}$ in both the training cohort (Additional file 2: Figure S1B-D) and validation cohort (Fig. $3 \mathrm{~b}-\mathrm{d})\left(\chi^{2} p<\right.$ 0.05). ProCUrE was able to differentiate low-risk vs intermediate- and high-risk PCa patients based on GS and CAPRA score, but not D'Amico criteria (Fig. 3b-d). Furthermore, ProCUrE's prognostic value was consistently more robust as demonstrated by a more stringent $p$ value $\left(\chi^{2} p<0.01\right)$ compared to age-adjusted PSA $\left(\chi^{2} p<0.05\right)$ in the validation cohort.

ProCUrE exhibited higher PPV than age-adjusted PSA for several different prognostic assessments (PPV: 76\% vs $70.5 \%$ for CAPRA, $76 \%$ vs $72.1 \%$ for D’Amico, and
$76 \%$ vs $63.9 \%$ for GS risk, Table 3B-D) indicating its overall robust ability to identify CS-PCa. Additionally, we found that ProCUrE significantly differentiated high-grade $\mathrm{PCa}$, including GS $\geq 7(4+3)$, and $\mathrm{GS} \geq 8$ from all other patients, with higher PPV (37.5\% and $31.3 \%$, respectively) compared to age-adjusted PSA (24.5\% and 16.7\%, respectively) (Fig. 3, Table 3B-D).

These results indicate that patients who are ProCUrE +ve have a higher likelihood of harboring CS-PCa and high-grade $(\mathrm{GS} \geq 8)$ tumors.

\section{Additional discriminative value of ProCUrE to PSA}

To determine whether ProCUrE could add discriminatory value to PSA testing, we performed concordance statistics (c-statistic) analysis of PSA alone and ProCUrE with PSA combined in the training cohort using logistic regression. The c-statistic with PSA and ProCUrE 

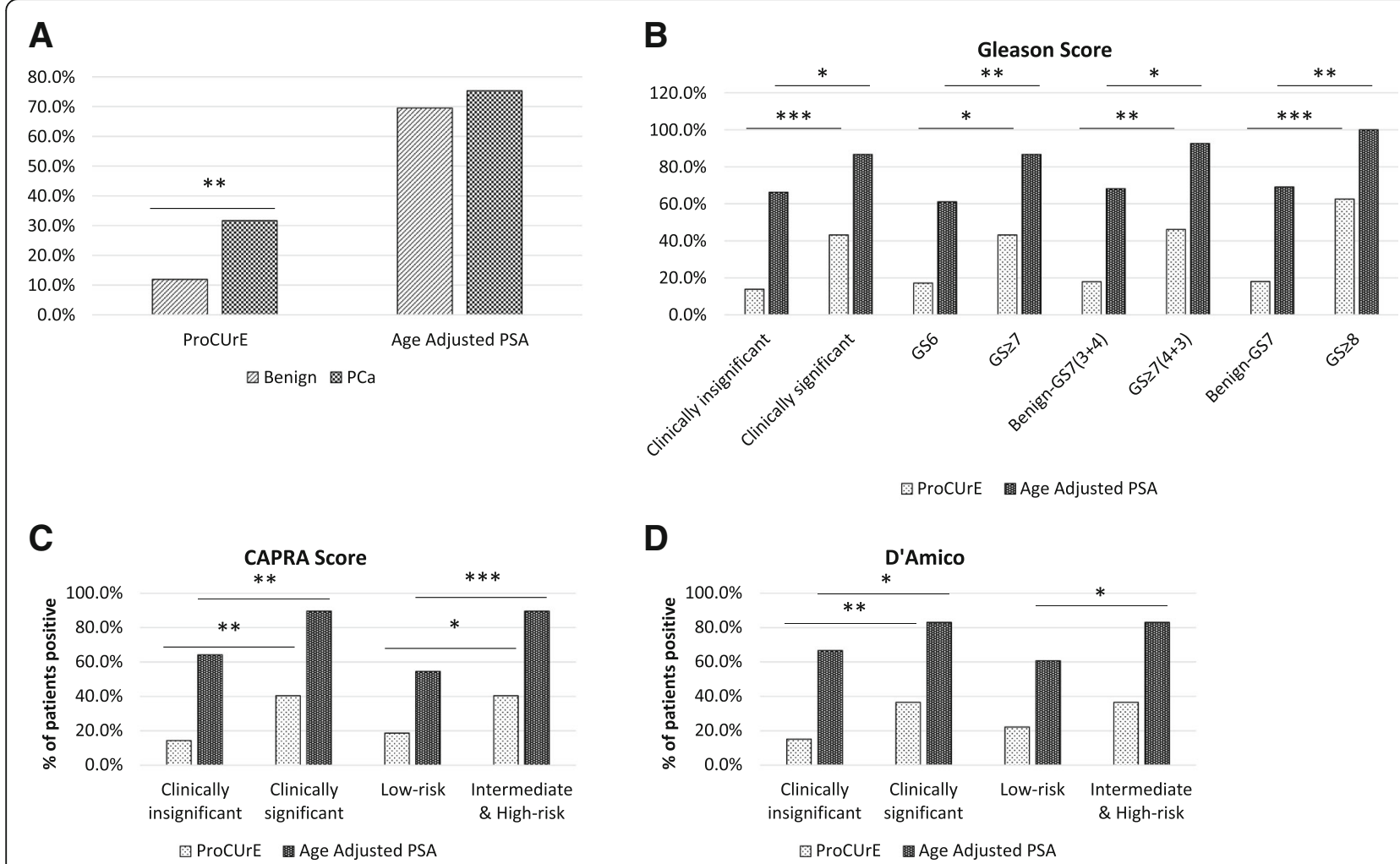

Fig. 3 Diagnostic and prognostic ability of ProCUrE and age-adjusted PSA in the validation cohort. a The percent false- and true-positive for ProCUrE or age-adjusted PSA separating benign and PCa patients. $\mathbf{b}$ The percent of patients positive for ProCUrE or age-adjusted PSA for clinically insignificant (benign and low-risk) vs clinically significant (intermediate- and high-risk) based on Gleason score. c, d The percent of patients positive for ProCUrE or age-adjusted PSA for clinically insignificant (benign and low-risk) vs clinically significant (intermediate- and highrisk) and low-risk vs intermediate- and high-risk as determined by CAPRA score and D'Amico. $N=140, x^{2 *} p<0.05,{ }^{* *} p<0.01,{ }^{* * *} p<0.001$

combined (0.775) is significantly improved over PSA alone (0.729) (DeLong test $p=0.039$ ), indicating that ProCUrE has additional discriminatory value to PSA for detecting CS-PCa (Table 5). Only GS risk was analyzed since CAPRA score and D'Amico criteria is calculated using PSA.

\section{Additional discriminative value of ProCUrE to PCPT}

To determine whether ProCUrE could add additional value to current clinical nomograms, we used the PCPT risk calculator for risk assessment in a subset of 144 patients (out of 408 patients in total) that had family history, ethnicity, and DRE results available.

We assessed the diagnostic (detection of any PCa) and prognostic (detection of $\mathrm{GS} \geq 7 \mathrm{PCa}$ ) value of PCPT (AUC $=0.741 ; 0.771$, respectively) and ProCUrE (AUC = 0.746; 0.730, respectively) individually. Further, using c-statistic, we calculated the additive value of ProCUrE to PCPT for diagnosis of $\mathrm{PCa}$ using logistic regression, which increased from $c=0.741$ for PCPT alone to $c=$ 0.817 for PCPT with ProCUrE. Similarly, for the detection of CS-PCa (as determined by GS) addition of ProCUrE to PCPT increased from $c=0.771$ to $c=0.822$.
Both values represent a significant increase (DeLong's test $p=0.011$ for diagnostic, $p=0.022$ for prognostic value) and indicate that the information provided by ProCUrE could further improve current PCPT parameters for prognosticating PCa patients prior to biopsy.

\section{Discussion}

Our study developed a urinary DNA methylation biomarker-based actionable assay, ProCUrE, to identify CS-PCa that would warrant treatment. ProCUrE significantly improves risk stratification with a higher PPV compared to age-adjusted PSA. Patients who are positive for ProCUrE will be more likely to harbor aggressive tumors and thus ProCUrE has the potential to supplement PSA or other tests that focus on NPV. Importantly, ProCUrE has additive value to PSA assessment and to PCPT risk calculator for the detection of aggressive (GS $\geq 7$ ) cancers.

PSA testing cannot reliably distinguish patients that have CS-PCa disease from those that do not require treatment. Therefore, invasive confirmation biopsy is necessary for $\mathrm{PCa}$ diagnosis and prognostication. A non-invasive adjunct test to PSA, such as ProCUrE, that 
Table 3 Diagnosis (A) and prognostication (B-D) of PCa

\begin{tabular}{|c|c|c|}
\hline & PPV & NPV \\
\hline \multicolumn{3}{|l|}{ A } \\
\hline \multicolumn{3}{|l|}{ Benign vs PCa } \\
\hline ProCUrE & $78.10 \%$ & $49.10 \%$ \\
\hline Age-adjusted PSA & $59.80 \%$ & $47.40 \%$ \\
\hline \multicolumn{3}{|l|}{ B } \\
\hline \multicolumn{3}{|c|}{ GS clinically insignificant vs clinically significant } \\
\hline ProCUrE & $59.40 \%$ & $76.40 \%$ \\
\hline Age-adjusted PSA & $38.20 \%$ & $84.20 \%$ \\
\hline \multicolumn{3}{|l|}{ GS6 vs GS $\geq 7$} \\
\hline ProCUrE & $76.00 \%$ & $53.70 \%$ \\
\hline Age-adjusted PSA & $63.90 \%$ & $70.00 \%$ \\
\hline \multicolumn{3}{|c|}{ Benign, GS6, GS7(3 + 4) vs GS $\geq 7(4+3)$} \\
\hline ProCUrE & $37.5 \%$ & $86.8 \%$ \\
\hline Age-adjusted PSA & $24.5 \%$ & $94.7 \%$ \\
\hline \multicolumn{3}{|c|}{ Benign, GS6, GS7 vs GS $\geq 8$} \\
\hline ProCUrE & $31.3 \%$ & $94.3 \%$ \\
\hline Age-adjusted PSA & $16.7 \%$ & $100.0 \%$ \\
\hline \multicolumn{3}{|l|}{ C } \\
\hline \multicolumn{3}{|c|}{ CAPRA clinically insignificant vs clinically significant } \\
\hline ProCUrE & $59.40 \%$ & $73.60 \%$ \\
\hline Age-adjusted PSA & $42.20 \%$ & $86.80 \%$ \\
\hline \multicolumn{3}{|c|}{ CAPRA low risk vs intermediate and high risk } \\
\hline ProCUrE & $76.00 \%$ & $48.10 \%$ \\
\hline Age-adjusted PSA & $70.50 \%$ & $75.00 \%$ \\
\hline \multicolumn{3}{|l|}{ D } \\
\hline \multicolumn{3}{|c|}{ D'Amico clinically insignificant vs clinically significant } \\
\hline ProCUrE & $59.40 \%$ & $68.90 \%$ \\
\hline Age-adjusted PSA & $43.10 \%$ & $76.30 \%$ \\
\hline \multicolumn{3}{|c|}{ D'Amico low risk vs intermediate and high risk } \\
\hline ProCUrE & $76.00 \%$ & $38.90 \%$ \\
\hline Age-adjusted PSA & $72.10 \%$ & $55.00 \%$ \\
\hline
\end{tabular}

Positive (PPV) and negative (NPV) predictive values for ProCUrE and ageadjusted PSA in the validation cohort separating benign vs PCa (A); clinically insignificant (benign and low-risk) vs clinically significant (intermediate- and high-risk) and low-risk vs clinically significant (intermediate- and high-risk) as determined by GS, CAPRA score, D'Amico criteria. $\left(x^{2} p\right.$ values for these comparisons could be found in Fig. 3)

can identify patients with CS-PCa would reduce overtreatment and prevent morbidity associated with unnecessary biopsies.

ProCUrE is comprised of the promoter methylation of HOXD3 and GSTP1 genes. HOXD3 is a member of the homeobox gene family of transcription factors which play important roles in morphogenesis and cell adhesion $[17,18]$, while GSTP1 is a member of the GST family of metabolic enzymes which function in regulation of cell cycle, DNA repair, and apoptosis [19]. Increased methylation levels of HOXD3 and GSTP1 are observed in prostate tumors and are correlated with aggressive PCa and/or adverse clinical outcomes [9, 18, 20, 21]. GSTP1 methylation has been previously investigated in urine sediments and was found to be PCa specific when compared to benign patients [22].

In a recent study of urinary methylation biomarkers, APC and GSTP1 methylation in conjunction with clinical variables demonstrated $100 \%$ NPV for distinguishing GS $\geq 7$ PCa [23]. Although this study demonstrated that urine-based DNA methylation markers could be used to prognosticate PCa aggressiveness, their results showed a high (26\%) false positive rate compared to only $13.8 \%$ false positive rate observed for ProCUrE. Thus, their combined panel of $A P C$ and GSTP1 is less than favorable to address the current challenges for managing $\mathrm{PCa}$, specifically, to minimize overtreatment of low-risk patients. Currently available non-invasive tests for $\mathrm{PCa}$ diagnosis and prognosis include the Prostate Health Index [24], SelectMDx [25], mpMRI [26], and PCA3. Similar to ProCUrE, SelectMDx is a post-DRE urine-based, two-gene (HOXC6 and DLX1) expression assay that can detect CS-PCa (GS >6) (AUC=0.77). The Prostate Health Index (PHI) [27] is a FDA-approved blood test that measures total, free and -2proPSA with greater specificity than free and total PSA for CS-PCa [28]. MpMRI has a high NPV (95\%) for GS $\geq 7$ tumors [26]. However, high cost and limited availability remain a limitation for implementing mpMRI as a screening tool. The Progensa PCA3 test is the only FDA approved urine-based test for PCa diagnosis. With its high NPV for PCa (90\%) [29], PCA3 can prevent unnecessary repeat biopsies. The Mi-Prostate score combines PCA3 and TMPRSS2:ERG fusion with the multivariable Prostate Cancer Prevention Trial risk calculator (PCPT) for prediction of $\mathrm{PCa}(\mathrm{AUC}=0.762)$ and high-risk $\mathrm{PCa}$ $(\mathrm{AUC}=0.779)$ which is comparable to our ProCUrE assay in the training cohort (AUC $=0.795$ for benign vs high-risk PCa, Fig. 2) [30] Additionally, we demonstrated that ProCUrE, when combined with PCPT, has even greater AUCs for diagnosis (0.817) and prognostication (0.822) of PCa than the Mi-Prostate score. However, it should be noted that this comparison was calculated on a subset of the total number of patients that had DRE, family history, and ethnicity information available.

All of the aforementioned tests are promising for $\mathrm{PCa}$ diagnosis or prognostication. However, all of these tests focus on NPV for PCa or high-risk PCa. Patients who are above the selected thresholds for these tests remain uncertain with respect to their disease status. Working in conjunction with the above tests or PSA, our ProCUrE assay fulfills a niche by focusing on PPV to offer a distinct advantage in identifying $\mathrm{PCa}$ patients with clinically significant tumors. Thus, patients who cannot be 
Table 4 Prediction of CS-PCa (as determined by GS) by individual markers, clinical variables, and ProCUrE

\begin{tabular}{|c|c|c|c|c|c|c|c|}
\hline \multicolumn{8}{|l|}{ A } \\
\hline Univariable & 1st quartile & 3rd quartile & Difference & OR & $95 \% \mathrm{Cl}$. & & $p$ value \\
\hline ProCUrE & -2.0006 & -1.0828 & 0.91789 & $1.58^{* * *}$ & 1.28 & 1.96 & $<0.0001$ \\
\hline APC & 0 & 0.37652 & 0.37652 & $1.24^{* * *}$ & 1.10 & 1.39 & 0.0003 \\
\hline HOXD3 & 0.43965 & 5.2043 & 4.7646 & $1.54^{* * *}$ & 1.24 & 1.90 & $<0.0001$ \\
\hline GSTP1 & 0 & 0.1216 & 0.1216 & $1.06^{* *}$ & 1.02 & 1.10 & 0.0013 \\
\hline KLK10 & 0 & 0.12665 & 0.12665 & $1.07^{* *}$ & 1.02 & 1.11 & 0.0048 \\
\hline TGF $\beta 2$ & 0 & 0.309 & 0.309 & $1.03^{*}$ & 1.00 & 1.06 & 0.0481 \\
\hline TBX15 & 0 & 0.21295 & 0.21295 & $1.16^{* * *}$ & 1.08 & 1.24 & $<0.0001$ \\
\hline PSA & 4.565 & 10.48 & 5.915 & $1.98^{* * *}$ & 1.46 & 2.68 & $<0.0001$ \\
\hline Age & 59 & 71 & 12 & $1.66^{* *}$ & 1.13 & 2.45 & 0.0105 \\
\hline PSA density & 0.09 & 0.2 & 0.11 & $2.35^{* * *}$ & 1.51 & 3.68 & 0.0002 \\
\hline Prostate volume & 40 & 70 & 30 & 0.70 & 0.47 & 1.06 & 0.0926 \\
\hline \multicolumn{8}{|l|}{ B } \\
\hline Multivariable & OR & $95 \% \mathrm{Cl}$. & & & $p$ value & & \\
\hline ProCUrE & $1.358^{*}$ & 1.051 & 1.754 & & 0.0194 & & \\
\hline PSA & 0.816 & 0.373 & 1.785 & & 0.6108 & & \\
\hline Age & $2.718^{* *}$ & 1.295 & 5.707 & & 0.0082 & & \\
\hline PSA density & $2.878^{* *}$ & 1.455 & 5.694 & & 0.0024 & & \\
\hline
\end{tabular}

Using univariable and multivariable logistic regression, the ability of individual methylation markers, ProCUrE, and clinical variables to differentiate CI-PCa and CSPCa as determined by GS was assessed in the training cohort. Since the scale of each variable is different, interquartile range odds ratios were estimated (logistic regression model ${ }^{*} p<0.05,{ }^{* *} p<0.01,{ }^{* *} p<0.001$ )

ruled out as having indolent tumors could be tested with ProCUrE to assess whether they have aggressive disease. ProCUrE could also be combined with tests such as SelectMDx to build a more comprehensive multivariable urine test in the future. This will improve current clinical $\mathrm{PCa}$ patient management once validated in independent studies.

Our study has certain limitations, including the fact that patient cohorts recruited for our study had differences in size and composition (e.g., UEA cohort had patients with higher PSA, GS, T stage compared to other cohorts) and as such, they could not be analyzed as three independent cohorts, despite using consensus recruitment criteria. Histopathological-based cancer diagnosis of biopsies was performed at three different participating centers which may have contributed to

Table 5 C-statistic for distinguishing clinically significant disease based on GS

\begin{tabular}{ll}
\hline Cl-PCa vs CS-PCa (GS) & C-statistic \\
\hline PSA & 0.729 \\
ProCUrE & 0.684 \\
Combined & $0.775^{*}$
\end{tabular}

C-statistics was used to determine any additive value of ProCUrE to PSA for discriminating CI-PCa vs CS-PCa as determined by GS in the training cohort. Only GS risk was analyzed since CAPRA score and D'Amico criteria is calculated using PSA. DeLong's test ${ }^{*} p=0.039$ some variation in Gleason grading between cohorts. However, our strategy of combining patients from all three cohorts and subsequently randomizing into training and validation cohorts, overcomes these caveats as it ensured that the training and validation sets would include patients representing a broad spectrum of prostate status, from benign with low PSA to very high risk PCa.

Other potential caveats are that benign patients with abnormalities such as high PSA may have contributed to the lack of significance for age-adjusted PSA with PCa diagnosis. Patients who are false positive for ProCUrE +ve status may actually harbor occult tumors. In this regard, follow-up data collection is ongoing for future biopsies and/or MRIs, which will enable assessment of ProCUrE for prediction and confirmation of CS-PCa. Additionally, clinical stage information for the UEA and Dublin cohorts did not differentiate T2a, T2b, and T2c tumors. Therefore, D'Amico criteria was calculated with all T2 patients assigned as intermediate risk. This may have contributed to the lack of significance for ProCUrE to stratify patients based on D'Amico criteria. In previously published studies, we described a 4-gene methylation Classifier Panel (APC, GSTP1, CRIP3, HOXD8) in PCa patients monitored by active surveillance (AS) for the prediction of risk-reclassification [12, 31]. We were unable to screen two genes (CRIP3 and HOXD8) from 
this four-gene classifier panel in the current study due to limitations of DNA samples availability. Similar to our findings in the AS PCa patient cohort, we found that methylation frequencies of $A P C$ and GSTP1 in urinary sediment were lower compared to those reported in tissue samples. Lastly, it is difficult to assess the additive value of ProCUrE to PSA for identifying patients based on the CAPRA score and D'Amico criteria, since both nomograms are calculated using PSA leading to strong association with PSA with these risk groups. Both CAPRA and D'Amico criteria are limited in that they require prostate biopsy to calculate risk. ProCUrE is advantageous in this regard since risk assessment can be performed prior to biopsy.

\section{Conclusion}

A non-invasive urine-based assay that can distinguish $\mathrm{PCa}$ patients with aggressive, clinically significant disease from those with benign and/or low risk disease would be valuable in reducing morbidity associated with over-diagnosis and preventing under-diagnosis of patients that would benefit from definitive treatment. Our ProCUrE assay could be used to supplement PSA screening and monitoring so those with aggressive disease would be identified early and those without will avoid unnecessary treatment.

\section{Materials and methods}

\section{Patient cohorts}

Participants were prospectively recruited between April 2012 and September 2015, from the University of East Anglia/Norfolk and Norwich University Hospital, UK (UEA cohort, $n=194$ ), the University Health Network, Canada (UHN cohort, $n=155$ ), and Trinity College, Ireland (Dublin cohort, $n=59$ ), together as part of the Movember GAP1 Multi-Center Urine Biomarker (MoGAP-MUB) cohort. Patients underwent prostate TRUS biopsy due to increased PSA and/or abnormal DRE (PSA follow-up time 0-122 months). Benign patients with normal age-adjusted PSA were recruited due to symptoms of $\mathrm{BPH}$ or had microhematuria detectable on dipstick only (i.e., not gross hematuria). Less than 10\% (39/408) patients had prior biopsies, all other patients were recruited at initial biopsy. Post-DRE first catch urine samples were either collected prior to biopsy or at least 1-month post-biopsy. Samples were mostly collected within 12 months from the date of biopsy. There were two patients that had > 12-month difference between biopsy and sample collection (range 14-146 months) and three patients with unknown biopsy dates. The patient with sample collected 146 months post-biopsy and all patients with unknown biopsy dates were benign patients. Informed consent was obtained following protocols approved by the research ethics boards of all centers and Sinai Health System, Toronto, Canada.

The cut-off for normal PSA (referred to as age-adjusted PSA) was determined following British Association of Urological Surgeon guidelines [15, 16]. Patients were classified based on the following criteria:

Benign indicates patients with negative biopsy.

GS, D’Amico criteria, and CAPRA score were utilized to stratify risk in $\mathrm{PCa}$ patients:

GS: low risk (GS $\leq 6)$, intermediate risk (GS7), and high risk $(\mathrm{GS} \geq 8)$.

D'Amico criteria: low risk (GS $\leq 6$ and T1-T2a and PSA $<10 \mathrm{ng} / \mathrm{mL}$ ), intermediate risk (GS7 or T2b or PSA $10-20 \mathrm{ng} / \mathrm{mL}$ ), and high risk (GS $\geq$ or $>$ or PSA $>20 \mathrm{ng} /$ $\mathrm{mL})$ [3].

The CAPRA score is defined as the sum of the following variables: age at diagnosis $(<50=0, \geq 50=1)$, PSA at diagnosis $(\mathrm{ng} / \mathrm{mL})(\leq 6=0,6.1-10=1,10.1-20=$ 2, 20.1-30 $=3,>30=4$ ), biopsy Gleason pattern (no pattern $\geq 4=0$, secondary pattern $\geq 4=1$, primary pattern $\geq 4=3$ ), clinical $\mathrm{T}$ stage ( $\mathrm{T} 1$ or $\mathrm{T} 2=0, \geq \mathrm{T} 3=1$; \%core: $<34 \%=0, \geq 34 \%=1$ ) [4]. CAPRA risk categories are as follows: low risk (0-2 points), intermediate risk $[3-5]$, and high risk $(\geq 6)$.

Calculation of PCPT risk score

PCPT risk was calculated using the Cleveland Clinic Risk Calculator Library - PCPT Risk Calculator v2.0 [5].

\section{Urine collection/processing}

Up to $50 \mathrm{~mL}$ of first catch urine was collected from each patient following DRE and centrifuged at $1200 \times g$ for $5 \mathrm{~min}$. Urine sediments were separated from supernatant and resuspended in $1 \mathrm{ml}$ of PBS and stored at $-80{ }^{\circ} \mathrm{C}$. Urinary sediment DNA was extracted using the AllPrep DNA/RNA mini-kit (Qiagen Inc.) Bisulfite conversion was as previously described [12].

\section{MethyLight analysis}

Multiplex MethyLight, a methylation-specific qPCR assay was used to determine the methylation levels of APC, GSTP1, HOXD3, KLK10, TBX15, and TGFB2 [32]. $A L U$-C4 $(A L U)$ was used as a methylation-independent, sodium bisulfite conversion-dependent internal input DNA control.

Primer/probe concentrations, cycling parameters, and data acquisition/analysis were as previously described, using Applied Biosystems 7500 (Life Technologies) [12].

Gene methylation was scored as percent methylated of reference (PMR) according to Eads et al. [33] CpGenome Universal Methylated DNA (EMD Millipore) was used as the positive control and to generate standard curves. Quality control criteria included genes of interest (GOIs) standard curve $R^{2}>0.95$, ALU $R^{2}>0.99$, and slope range from -3.28 to -4.86 . Any sample with a 
higher cycling threshold (lower quantity) for $A L U$ than the least concentrated standard curve point for which all GOI amplified was excluded from analysis. Samples were analyzed in duplicate and were reanalyzed if replicates had a difference in PMR of $>10 \%$. Data development and analysis were carried out in accordance with the Minimum Information for Publication of Quantitative real-time PCR Experiments (MIQE) guideline [34].

\section{Calculation for ProCUrE}

Least absolute shrinkage and selection operator (LASSO) was applied to construct gene models using benign vs CAPRA high-risk (CAPRA-HR) patients in the training cohort. LASSO was used to eliminate genes that had insufficient contribution to the model. The remaining genes (APC, GSTP1, HOXD3, KLK10, TGF 2 ) with non-zero coefficients as determined by LASSO were tested for every possible combination using the generalized linear model in the training cohort to determine their AUC, Akaike information criterion (AIC), and Bayesian information criterion (BIC). An optimal two-gene model consisting of HOXD3 and GSTP1, which had the highest AUC with the lowest AIC and BIC, was selected for further analysis. We developed Prostate Cancer Urinary Epigenetic (ProCUrE) assay, based on the formula:

$$
\begin{aligned}
& \text { Intercept }+ \text { Coefficient }(\text { GSTP1 }) * \text { PMR(GSTP1) } \\
& + \text { Coefficient }(\text { HOXD3 }) * \text { PMR }(\text { HOXD3 })
\end{aligned}
$$

where the intercept is -0.8395549 , the coefficient for HOXD3 is 0.1397128 , and the coefficient for GSTP1 is 0.8632709 .

Additional comparisons (benign vs $\mathrm{PCa}, \mathrm{CI}$ vs CS-PCa as determined by GS, CAPRA and D'Amico) were performed in the training cohort (Additional file 2: Figure S2). However, none of these comparisons yielded a model with as robust discriminative value (higher AUC) as observed with benign vs CAPRA-HR comparison. Therefore, we opted for the model constructed using benign vs CAPRA-HR for further analysis.

\section{Statistical analysis}

Spearman's $\rho$ rank was used to compare PMR, age, \%core, prostate volume (cc), and PSA at diagnosis. ROC curve analysis was used to determine ProCUrE's sensitivity and specificity at every cut-off value. The value with the highest sum of sensitivity and specificity was chosen as the optimized threshold. The same numerical values derived from the training cohort were used in the validation cohort: threshold derived from ROC analysis (threshold value $=0.574264899821094)$ and intercept and coefficients derived from generalized linear modeling. $X^{2}$ tests were used to determine any significant association with overall cancer status or CS-PCa ( $\geq$ intermediate-risk cancer as determined by GS, CAPRA, or D'Amico criteria).

Univariable and multivariable logistic regression was performed to estimate odds ratios and corresponding 95\% confidence intervals to assess the ability of individual markers, ProCUrE, and clinical variables to identify CS-PCa patients using the lrm function of the "rms" R package (5.1-2). C-statistic was calculated using ROC curves [35]. DeLong's test [36] was used to compare significance for c-statistic as part of the roc.test function of the pROC R package (v1.13.0).

LASSO analysis was carried out using the "glmnet" function of the "glmnet" R package (v2.0-13) [37] to determine the optimal value of the penalty coefficient lambda, with 10 -fold cross-validation performed using the "cv.glmnet" function. Optimal lambda was chosen as the cross-validated lambda at the minimum binomial deviance. Model assessment was performed using the "ROCR" R package, AUC was determined via bootstrapping with 1000 iterations.

For all described methods, two-sided $p$ values of $<0.05$ were considered significant. All tests were conducted with IBM SPSS software (SPSS Inc. Released 2014. PASW Statistics for Windows, Version 22.0) or R version 3.4.0 [38]. Reporting recommendations for tumor marker prognostic studies (REMARK) guidelines were followed in analysis [39].

\section{Additional files}

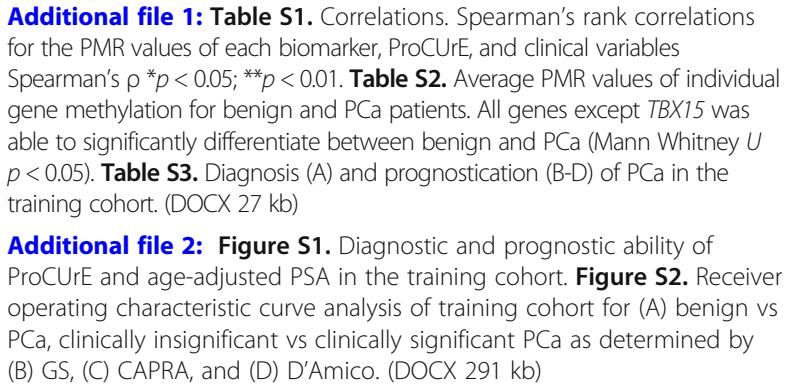

Additional file 1: Table S1. Correlations. Spearman's rank correlations for the PMR values of each biomarker, ProCUrE, and clinical variables Spearman's $\rho{ }^{*} p<0.05 ;{ }^{* *} p<0.01$. Table S2. Average PMR values of individual gene methylation for benign and PCa patients. All genes except TBX15 was able to significantly differentiate between benign and PCa (Mann Whitney $U$ $p<0.05$ ). Table S3. Diagnosis (A) and prognostication (B-D) of PCa in the training cohort. (DOCX $27 \mathrm{~kb}$ )

Additional file 2: Figure S1. Diagnostic and prognostic ability of ProCUrE and age-adjusted PSA in the training cohort. Figure S2. Receiver operating characteristic curve analysis of training cohort for (A) benign vs PCa, clinically insignificant vs clinically significant PCa as determined by (B) GS, (C) CAPRA, and (D) D'Amico. (DOCX $291 \mathrm{~kb}$ )

\section{Abbreviations}

\%core: Percent of cores positive in biopsy; AIC: Akaike information criterion; AS: Active surveillance; AUC: Area under curve; BIC: Bayesian information criterion; CAPRA: Cancer of the Prostate Risk Assessment; CI-PCa: Clinically insignificant prostate cancer; CS-PCa: Clinically significant prostate cancer; DRE: Digital rectal exam; GAP: Global Action Plan; GOI: Gene of interest; GS: Gleason score; IQR OR: Interquartile range odds ratios; LASSO: Least absolute shrinkage and selection operator; MIQE: Minimum Information for Publication of Quantitative real-time PCR Experiments; MoGAPMUB: Movember GAP1 Multi-Center Urine Biomarker; MpMRI: Multiparametric magnetic resonance imaging; NPV: Negative predictive value; PCa: Prostate cancer; PCPT: Prostate Cancer Prevention Trial; PMR: Percent methylated of reference; PPV: Positive predictive value; ProCUrE: Prostate Cancer Urinary Epigenetic; PSA: Prostate specific antigen; ROC: Receiver operating characteristic; USPSTF: U.S. Preventive Services Task Force 


\section{Acknowledgements}

We would like to acknowledge the Movember Urine Biomarker Consortium which brought together 12 research teams from seven different countries to collaborate on such an important project. A list of members is provided below.

Movember GAP1 Urine Biomarker Collaborative Members

1. Colin Cooper, Jeremy Clark, Schools of Medicine and Biologica Sciences, University of East Anglia: Norwich, Norfolk. NR4 7TJ, UK.

2. Bharati Bapat, Lunenfeld-Tanenbaum Research Institute, Sinai Health Systems, University of Toronto, Toronto, Ontario, M5T 3L9, Canada.

3. Rob Bristow, Ontario Cancer Institute, Princess Margaret Hospital, Toronto, Ontario, Canada.

4. Chris Parker, Royal Marsden Hospital, Downs Road, Sutton, SM2 5PT,

5. Ian Mills, Queen's University Belfast. School of Medicine, Dentistry and Biomedical Sciences. Institute for Health Sciences, Belfast, UK.

6. Hardev Pandha, University of Surrey: Guildford, Surrey, GU2 7XH. UK.

7. Hayley Whitaker, Molecular Diagnostics and Therapeutics Group, Cruciform Building, University College London, Gower Street. WC1E 6BT. UK.

8. David Neal, David Neal, Cancer Research UK Cambridge Research Institute, Robinson Way, Cambridge, CB2 ORE. UK

9. Mireia Olivan, Andreas Doll, Vall d'Hebron Research Institute and Hospital and Autonomous University of Barcelona: 08035 Barcelona, Spain.

10. Hing Leung, The Beatson Institute for Cancer Research, Joseph Black Building, University of Glasgow, Glasgow, G12 8QQ, UK.

11. Antoinette Perry, Cancer Biology and Therapeutics Laboratory, School of Biomolecular and Biomedical Science, Conway Institute, University College Dublin, Dublin 4, Ireland.

12. Martin Sanda, Department of Urology, Winship Cancer Institute, Emory University School of Medicine, Atlanta, GA, USA.

13. Jack Schalken, Nijmegen Medical Centre, Radboud University, The Netherlands. Nijmegen, The Netherlands

We gratefully acknowledge the patients from three international centers who participated in this study. We also acknowledge assistance of staff at The UHN Genito-Urinary BioBank (GUBioBank) (Toronto). The UHN GUBioBank is a REB approved investigator-initiated bio-banking program that collects and archives biological specimens and data obtained from consenting urologic oncology patients. The purpose of the program is to facilitate the discovery and validation of novel biomarkers, which ultimately will enable advances in the area of personalized medicine in Urology.

\section{Funding}

This work was supported by grant funding from Movember (GAP1 Urine Biomarker Award), Prostate Cancer Canada No. 2011-700, Movember PCC TAG No. 2014-01 (B. Bapat), The Ontario Student Opportunity Trust Funds Award (F. Zhao, S. Kamdar, R. Jeyapala), and Ontario Graduate Scholarships (F. Zhao, E. Olkhov-Mitsel, S. Kamdar).

\section{Availability of data and materials}

The datasets used and/or analyzed during the current study are available from the corresponding author on reasonable request.

\section{Authors' contributions}

$\mathrm{BB}, \mathrm{JC}$, and NF contributed to the conception and design. FZ, EO-M, JG, NF, $J C, R H, M H, R M, A P, A T, E O ' R$, and SK took part in the acquisition of the data. $F Z$, EO-M, SK, RJ, DB, and BB were responsible for the analysis and interpretation of the data. FZ and BB drafted the manuscript. BB, EO-M, CC, DB, and $J C$ did the critical revision of the manuscript for important intellectual content. FZ, EO-M, SK, and DB contributed to the statistical analysis. BB and CC obtained the funding. $\mathrm{BB}, \mathrm{DB}, \mathrm{JC}, \mathrm{NF}$, and AP gave administrative, technical, or material support. BB did the supervision. All authors read and approved the final manuscript.

\section{Ethics approval and consent to participate}

Informed consent was obtained following protocols approved by the research ethics boards of all centers and Sinai Health System, Toronto, Canada.

\section{Consent for publication}

Informed consent was obtained from all participants at all centers.

\section{Competing interests}

The authors declare that they have no competing interests.

\section{Publisher's Note}

Springer Nature remains neutral with regard to jurisdictional claims in published maps and institutional affiliations.

\section{Author details}

'Lunenfeld-Tanenbaum Research Institute, Sinai Health System, Toronto, Canada. ${ }^{2}$ Department of Laboratory Medicine \& Pathobiology, University of Toronto, Toronto, Canada. ${ }^{3}$ Schools of Medicine and Biological Sciences, University of East Anglia, Norwich, Norfolk, UK. ${ }^{4}$ Norfolk and Norwich University Hospital, Norwich, Norfolk, UK. ${ }^{5}$ Cancer Biology and Therapeutics Laboratory, School of Biomolecular and Biomedical Science, Conway Institute, University College Dublin, Dublin 4, Ireland. ${ }^{6}$ The Earlham Institute, Norwich, Norfolk, UK. Division of Urology, University Health Network, University of Toronto, Toronto, Canada.

Received: 14 August 2018 Accepted: 28 October 2018

Published online: 23 November 2018

\section{References}

1. Force USPST. Final recommendation statement: prostate cancer. Screening 2012; [updated November 2013. Available from: https://www. uspreventiveservicestaskforce.org/Page/Name/second-annual-report-tocongress-on-high-priority-evidence-gaps-for-clinical-preventive-services.

2. Bhindi B, Mamdani M, Kulkarni GS, Finelli A, Hamilton RJ, Trachtenberg J, et al. Impact of the U.S. Preventive Services Task Force recommendations against prostate specific antigen screening on prostate biopsy and cancer detection rates. J Urol. 2015;193(5):1519-24.

3. D'Amico AV, Whittington R, Malkowicz SB, Schultz D, Blank K, Broderick GA, et al. Biochemical outcome after radical prostatectomy, external beam radiation therapy, or interstitial radiation therapy for clinically localized prostate cancer. JAMA. 1998;280(11):969-74.

4. Cooperberg MR, Hilton JF, Carroll PR. The CAPRA-S score: a straightforward tool for improved prediction of outcomes after radical prostatectomy. Cancer. 2011;117(22):5039-46.

5. Thompson IM, Ankerst DP, Chi C, Goodman PJ, Tangen CM, Lucia MS, et al. Assessing prostate cancer risk: results from the Prostate Cancer Prevention Trial. J Natl Cancer Inst. 2006;98(8):529-34.

6. Klotz L, Vesprini D, Sethukavalan $P$, Jethava V, Zhang L, Jain S, et al. Longterm follow-up of a large active surveillance cohort of patients with prostate cancer. J Clin Oncol. 2015;33(3):272-7.

7. Ahmad AS, Vasiljevic N, Carter P, Berney DM, Moller H, Foster CS, et al. A novel DNA methylation score accurately predicts death from prostate cancer in men with low to intermediate clinical risk factors. Oncotarget. 2016;7(44):71833-40.

8. Vasiljevic N, Wu K, Brentnall AR, Kim DC, Thorat MA, Kudahetti SC, et al. Absolute quantitation of DNA methylation of 28 candidate genes in prostate cancer using pyrosequencing. Dis Markers. 2011;30(4):15161.

9. Liu L, Kron KJ, Pethe W, Demetrashvili N, Nesbitt ME, Trachtenberg J, et al. Association of tissue promoter methylation levels of APC, TGFB2, HOXD3 and RASSF1A with prostate cancer progression. Int J Cancer. 2011;129(10): 2454-62.

10. Kron K, Liu L, Trudel D, Pethe V, Trachtenberg J, Fleshner N, et al. Correlation of ERG expression and DNA methylation biomarkers with adverse clinicopathologic features of prostate cancer. Clin Cancer Res. 2012; 18(10):2896-904.

11. Olkhov-Mitsel E, Van der Kwast T, Kron KJ, Ozcelik H, Briollais L, Massey C, et al. Quantitative DNA methylation analysis of genes coding for kallikreinrelated peptidases 6 and 10 as biomarkers for prostate cancer. Epigenetics. 2012;7(9):1037-45

12. Zhao F, Olkhov-Mitsel E, van der Kwast T, Sykes J, Zdravic D, Venkateswaran $\checkmark$, et al. Urinary DNA methylation biomarkers for noninvasive prediction of aggressive disease in patients with prostate cancer on active surveillance. J Urol. 2017;197(2):335-41. 
13. Dobbin KK, Simon RM. Optimally splitting cases for training and testing high dimensional classifiers. BMC Med Genet. 2011;4:31.

14. Dupuy A, Simon RM. Critical review of published microarray studies for cancer outcome and guidelines on statistical analysis and reporting. J Natl Cancer Inst. 2007;99(2):147-57.

15. Surgeons TBAoU. PSA MEASUREMENTS Frequently-Asked Questions [Available from: http://www.tsft.nhs.uk/media/45224/PSA_levels.pdf. Accessed 15 Oct 2018

16. DeAntoni EP, Crawford ED, Oesterling JE, Ross CA, Berger ER, McLeod DG, et al. Age- and race-specific reference ranges for prostate-specific antigen from a large community-based study. Urology. 1996;48(2): 234-9.

17. Hamada J, Omatsu T, Okada F, Furuuchi K, Okubo Y, Takahashi Y, et al. Overexpression of homeobox gene HOXD3 induces coordinate expression of metastasis-related genes in human lung cancer cells. Int J Cancer. 2001; 93(4):516-25.

18. Kron KJ, Liu L, Pethe W, Demetrashvili N, Nesbitt ME, Trachtenberg J, et al. DNA methylation of HOXD3 as a marker of prostate cancer progression. Lab Investig. 2010:90(7):1060-7.

19. Tew KD, Manevich Y, Grek C, Xiong Y, Uys J, Townsend DM. The role of glutathione S-transferase $\mathrm{P}$ in signaling pathways and S-glutathionylation in cancer. Free Radic Biol Med. 2011;51(2):299-313.

20. Jeronimo C, Usadel H, Henrique R, Silva C, Oliveira J, Lopes C, et al. Quantitative GSTP1 hypermethylation in bodily fluids of patients with prostate cancer. Urology. 2002;60(6):1131-5.

21. Wu T, Giovannucci E, Welge J, Mallick P, Tang WY, Ho SM. Measurement of GSTP1 promoter methylation in body fluids may complement PSA screening: a meta-analysis. Br J Cancer. 2011;105(1):65-73.

22. Goessl C, Muller M, Heicappell R, Krause H, Straub B, Schrader M, et al. DNAbased detection of prostate cancer in urine after prostatic massage. Urology. 2001;58(3):335-8.

23. Jatkoe TA, Karnes RJ, Freedland SJ, Wang Y, Le A, Baden J. A urine-based methylation signature for risk stratification within low-risk prostate cancer. Br J Cancer. 2015;112(5):802-8.

24. Loeb S, Catalona WJ. The Prostate Health Index: a new test for the detection of prostate cancer. Ther Adv Urol. 2014;6(2):74-7.

25. Leyten GH, Hessels D, Smit FP, Jannink SA, de Jong H, Melchers WJ, et al. Identification of a candidate gene panel for the early diagnosis of prostate cancer. Clin Cancer Res. 2015;21(13):3061-70

26. Ahmed HU, El-Shater Bosaily A, Brown LC, Gabe R, Kaplan R, Parmar MK, et al. Diagnostic accuracy of multi-parametric MRI and TRUS biopsy in prostate cancer (PROMIS): a paired validating confirmatory study. Lancet. 2017; 389(10071):815-22.

27. White J, Shenoy BV, Tutrone RF, Karsh LI, Saltzstein DR, Harmon WJ, et al. Clinical utility of the Prostate Health Index (phi) for biopsy decision management in a large group urology practice setting. Prostate Cancer Prostatic Dis. 2018:21(1):78-84

28. Nordstrom T, Vickers A, Assel M, Lilja H, Gronberg H, Eklund M. Comparison between the four-kallikrein panel and prostate health index for predicting prostate cancer. Eur Urol. 2015;68(1):139-46.

29. Gittelman MC, Hertzman B, Bailen J, Williams T, Koziol I, Henderson RJ, et al. PCA3 molecular urine test as a predictor of repeat prostate biopsy outcome in men with previous negative biopsies: a prospective multicenter clinical study. J Urol. 2013;190(1):64-9.

30. Tomlins SA, Day JR, Lonigro RJ, Hovelson DH, Siddiqui J, Kunju LP, et al. Urine TMPRSS2:ERG plus PCA3 for individualized prostate cancer risk assessment. Eur Urol. 2016;70(1):45-53.

31. Bapat B. Re: urinary DNA methylation biomarkers for noninvasive prediction of aggressive disease in patients with prostate cancer on active surveillance: F. Zhao, E. Olkhov-Mitsel, T. van der Kwast, J. Sykes, D. Zdravic, V. Venkateswaran, A. R. Zlotta, A. Loblaw, N. E. Fleshner, L. Klotz, D. Vesprini and B. Bapat. J Urol 2017;197:335-341. J Urol. 2018. https://doi.org/10.1016/j.juro.2017.10.061

32. Olkhov-Mitsel E, Zdravic D, Kron K, van der Kwast T, Fleshner N, Bapat B. Novel multiplex MethyLight protocol for detection of DNA methylation in patient tissues and bodily fluids. Sci Rep. 2014:4:4432.

33. Eads CA, Danenberg KD, Kawakami K, Saltz LB, Blake C, Shibata D, et al. MethyLight: a high-throughput assay to measure DNA methylation. Nucleic Acids Res. 2000;28(8):E32
34. Bustin SA, Benes V, Garson JA, Hellemans J, Huggett J, Kubista M, et al. The MIQE guidelines: minimum information for publication of quantitative realtime PCR experiments. Clin Chem. 2009;55(4):611-22.

35. Steyerberg EW. Clinical prediction models a practical approach to development, validation, and updating. New York: Springer; 2009.

36. DeLong ER, DeLong DM, Clarke-Pearson DL. Comparing the areas under two or more correlated receiver operating characteristic curves: a nonparametric approach. Biometrics. 1988;44(3):837-45.

37. Friedman J, Hastie T, Tibshirani R. Regularization paths for generalized linear models via coordinate descent. J Stat Softw. 2010;33(1):1-22.

38. TR Core Team. R: A language and environment for statistical computing. R Foundation for Statistical Computing, Vienna, Austria. 2014. http://www.Rproject.org/.

39. McShane LM, Altman DG, Sauerbrei W, Taube SE, Gion M, Clark GM, et al. Reporting recommendations for tumor marker prognostic studies. J Clin Oncol. 2005;23(36):9067-72.

\section{Ready to submit your research? Choose BMC and benefit from:}

- fast, convenient online submission

- thorough peer review by experienced researchers in your field

- rapid publication on acceptance

- support for research data, including large and complex data types

- gold Open Access which fosters wider collaboration and increased citations

- maximum visibility for your research: over $100 \mathrm{M}$ website views per year

At BMC, research is always in progress.

Learn more biomedcentral.com/submissions 